\title{
The Neonatal Salivary Transcriptome
}

\author{
Jill L. Maron \\ Department of Pediatrics, Mother Infant Research Institute, Tufts Medical Center, Boston, Massachusetts 02111 \\ Correspondence: jmaron@tuftsmedicalcenter.org
}

The ability to noninvasively assess the physical and developmental status of a neonate is a goal of modern medicine. In recent years, technological advances have permitted the highthroughput analysis of saliva for thousands of genes, proteins, and metabolites from a single sample source. Saliva is an ideal biofluid to assess health, disease, and development in the newborn. It may be harnessed repeatedly, even in the most vulnerable patients, without risk of harm. Translating novel information about an infant's global development and risk of disease to the neonatal bedside through the salivary transcriptome has the potential to significantly improve clinical care and outcomes in this at-risk population.

$\mathrm{N}_{\mathrm{t} \text { ture }}^{\mathrm{e}}$ eonates, particularly those born premature, present unique challenges to caregivers. Their small size, clinical fragility, and limited blood volumes often prohibit invasive testing and may limit enrollment into clinical trials aimed at improving care through the development and implementation of novel treatment strategies. Thus, utilizing a noninvasive and easily accessible biofluid that provides caregivers with essential information about the health status and development of a newborn holds great promise for significantly improving patient care and outcomes (Bianchi et al. 2010).

Saliva is a diverse source of genetic material (DNA and RNA), proteins, metabolites, and microorganisms. Through both extracellular and intracellular trafficking mechanisms, biomarkers enter into the oral cavity and may be monitored to inform an investigator about overall health and systemic disease in a patient. Saliva has the benefits over other noninvasively obtained biofluids, such as urine or stool, in that it may be obtained on demand and repeatedly in a limited time frame. These advantages make it an ideal biofluid for noninvasive clinical assessment across a wide variety of healthcare settings, including in the vulnerable newborn population (Wong 2012; Schafer et al. 2014).

Although salivary studies identifying single proteins (e.g., cortisol) (Maganano et al. 1992) or microorganisms (e.g., cytomegalovirus) (Balcarek et al. 1993) in the newborn were performed more than 20 years ago, genomic, transcriptomic, and proteomic salivary analyses are relatively new fields of discovery. Arguably, the vast majority of preterm infants are born without genetic syndromes. Rather, their unique phenotypes, diseases, and developmental impairments are a consequence of the effects of preterm birth on transcriptional and translational processes and epigenetic modifications. Gaining access, in real-time, to ongoing disrupted developmental patterns at a molecular level, particularly at the level of the transcriptome,

Editors: Diana W. Bianchi and Errol R. Norwitz

Additional Perspectives on Molecular Approaches to Reproductive and Newborn Medicine available

at www.perspectivesinmedicine.org

Copyright (C) 2016 Cold Spring Harbor Laboratory Press; all rights reserved; doi: 10.1101/cshperspect.a026369

Cite this article as Cold Spring Harb Perspect Med 2016;6:a026369 
may allow caregivers an unprecedented opportunity to identify infants at risk for specific neonatal systemic and/or developmental sequelae. Utilizing saliva to further our understanding of complex transcriptional regulatory mechanisms may also identify novel targets for intervention and therapy for those born premature.

This review will highlight the recent emergence of the salivary transcriptome as a novel and noninvasive means to access real-time genetic information to assess clinical status and disease susceptibility. Specific emphasis will be placed on the diagnostic and potentially prognostic potential of salivary transcriptomics in the premature newborn population and focus on the enormous amount of developmental information available to caregivers from a single sample source. It will address unique challenges associated with salivary analysis in the newborn and review alternative strategies to ensure that these techniques translate to neonatal care. Finally, it will highlight specific applications of salivary transcriptomics in the premature newborn in which ongoing assessment of developmental skills may not only improve short- and long-term patient care and outcomes but also reduce health care costs.

\section{SALIVA AS A BIOFLUID}

Despite its reputation as a rich source of biomarkers, the vast majority of saliva is water. Only $1 \%$ of the volume contains a mixture of electrolytes, microorganisms, proteins, and genetic material. Ninety percent of saliva is formed in the major salivary glands (parotid, submandibular, and sublingual), with $10 \%$ forming in the minor glands (labial, buccal, and palatal) (Cuevas-Córdoba and Santiago-García 2014). As a direct filtrate of blood, saliva shares many of the same biomarkers found in plasma. Studies in adults comparing the proteomic profiles of plasma and serum from the same individuals revealed a $27 \%$ similarity between biofluids (Loo et al. 2010). Similarly, comparative transcriptomic analyses between umbilical cord blood and neonatal saliva revealed $\sim 40 \%$ similarity in genes expressed at the highest quintile in each biofluid (Maron et al. 2012a). Saliva is also a rich source of microorganisms, including viruses, bacteria, and fungi, whose composition may serve as a window into the overall health status of an individual. Comprehensive reviews of adult saliva have been previously written and can be found elsewhere (Lawrence 2002; LlenaPuy 2006; Tiwari 2011; Cuevas-Córdoba and Santiago-García 2014).

The saliva of a newborn differs from its adult counterpart because of several distinct characteristics. First, newborns lack teeth. Because a large proportion of microorganisms in the oral cavity are located near teeth, the microbiotic component of a newborn's saliva differs from adults and older children (Pennisi 2005). Second, colonization with microorganisms in the oropharynx occurs rapidly in the days to weeks following birth. Premature newborns are often delivered via cesarean section, may not feed for days, and have instrumentation with nonsterile nasogastric and endotracheal tubes that may lead to additional disruption of microbiotic colonization. Finally, there have been few, if any, studies conducted that examine the impact that the developing salivary glands may have on salivary constituents in the human newborn. It is possible that filtration and diffusion mechanisms are affected by the ongoing development of each gland, further altering salivary substrates in a neonate. Although these effects may not influence data obtained on neonatal subjects, care should be made when extrapolating adult saliva studies to this population and/ or combining samples obtained from different developmental stages.

\section{SALIVARY COLLECTION, STORAGE AND PROCESSING}

Historically, RNA's single stranded structure, along with the oropharynx's ubiquitous RNAses, had made RNA highly susceptible to rapid degradation. However, for over a decade, commercially available salivary collection kits have made it possible for investigators to interrogate both messenger RNA (mRNA) and regulatory or microRNA (miRNA) in saliva samples. An essential first step to salivary transcriptomics is the rapid stabilization of the sample by inhibit- 
ing RNAses, halting gene expression changes and limiting microbial overgrowth within minutes of salivary collection. Comparative studies analyzing the effectiveness of varying salivary stabilizing solutions for downstream transcriptomic analysis have been performed. Wong (2007) reported that RNALater is a poor stabilizing reagent because of its high salt content and ability to increase cellular membrane permeability, which increases the release of contaminating genomic DNA. In 2011, Lee et al. (2011) described a direct saliva transcriptome analysis that successfully used an ambient-temperature processing, stabilization and storage protocol for salivary RNA. More recently, Pandit and colleagues (2013) have modified an existing Qiagen protocol (QIAzol), to provide investigators with a robust, cost-effective alternative to other commercially available RNA extraction kits. By comparison, published reports in the newborn have successfully used Qiagen's RNAProtect Saliva Solution on multiple downstream transcriptomic platforms (Maron et al. 2010, 2012b; Dietz et al. 2012). Thus, investigators have options when considering salivary collection and extraction kits and should consider study design, location and costs when choosing a product or protocol.

Collection of saliva samples in the newborn poses additional challenges to the investigator. In older children and adults, subjects can provide passive drool or use appropriate sponges or collection devices for saliva collection. Further, subjects can rinse their mouths out before sample collection to limit food or debris contami- nation. However, in the newborn, samples must be obtained by the investigator. Thus, there is a theoretical risk of human breast milk contamination in neonatal saliva samples that may alter their gene expression profiles. Dietz and colleagues (2012) have developed an easy to use system that relies on readily available equipment found in most neonatal intensive care units (NICU) and special care nursery settings. Briefly, saliva is collected with a $1 \mathrm{~mL}$ syringe, end caps removed, attached to low-wall suction. Saliva is collected in the syringe for $20-30 \mathrm{sec}$ and immediately stabilized at the bedside. Samples may then be left at room temperature, $4^{\circ} \mathrm{C}$ or frozen at $-20^{\circ} \mathrm{C}$ or $-80^{\circ} \mathrm{C}$ pending processing and manufacturer's guidelines (Dietz et al. 2012). Most commercially available collection kits allow for samples to sit at room temperature for days to weeks without any risk of RNA degradation. Sponges are also available for saliva collection in the newborn, but investigators must take the additional step of centrifugation to retrieve the saliva sample. It should be noted that in the neonatal population, modifications to existing protocols might need to be made to account for the limited salivary volumes available (Table 1) (Maron 2011; Dietz et al. 2012). Most salivary collection kits recommend $\geq 1 \mathrm{~mL}$ of saliva for downstream RNA extraction and analysis. However, neonates on average can only generate $\leq 50 \mu \mathrm{L}$ of saliva per sample (Maron et al. 2010, 2012b). Despite these small volumes, successful extraction and identification of RNA targets has been performed repeatedly in the newborn. Thus, investigators

Table 1. Unique considerations for salivary analysis in the newborn

\begin{tabular}{|c|c|c|}
\hline & Adults & Neonates \\
\hline Salivary volume yield & Average collection: $1.5-2 \mathrm{~mL}$ & Average collection: $10-50 \mu \mathrm{L}$ \\
\hline \multirow[t]{3}{*}{ Collection devices } & Voluntary passive drool & Sponges \\
\hline & Sponges & Suction catheters \\
\hline & & Filter paper \\
\hline $\begin{array}{l}\text { Contamination } \\
\text { considerations }\end{array}$ & $\begin{array}{l}\text { Limit food and/or drink before salivary } \\
\text { collection }\end{array}$ & $\begin{array}{l}\text { Potential for human breast milk } \\
\text { contamination }\end{array}$ \\
\hline & Rinse mouth before salivary collection & \\
\hline Processing & $\begin{array}{l}\text { Multiple available processing protocols } \\
\text { available (Wong 2007; Lee et al. 2011; } \\
\text { Pandit et al. 2013) }\end{array}$ & $\begin{array}{l}\text { Modifications may be required because of } \\
\text { smaller starting volumes (Dietz et al. } \\
\text { 2012) }\end{array}$ \\
\hline
\end{tabular}


J.L. Maron

should not be deterred by the limited starting saliva volumes or the required protocol modifications when designing studies for the newborn (Table 1).

\section{SALIVARY RNA AND DOWNSTREAM APPLICATIONS}

With the availability of RNA stabilizing reagents, the first reports of the detection, identification and diagnostic application of salivary RNAs began emerging. In their original article, Li et al. (2004a) described the identification of salivary mRNAs in healthy adult subjects. The investigators performed whole transcriptomic microarray analyses on saliva samples collected from 10 subjects (six males) and identified what they coined as the Normal Core Salivary Transcriptome (NCST) (Li et al. 2004a). NCST is composed of 185 genes expressed in all study subjects. Reverse transcription-quantitative polymerase chain reaction (RT-qPCR) studies confirmed the universal expression of each gene in adult saliva samples. Similarly, Maron et al. (2012a) reported on the neonatal saliva transcriptome and described genes expressed at the highest quintile on gene expression microarrays in all term neonatal study subjects $(\mathrm{n}=15)$. Comparative analysis between both published adult and neonatal gene lists by Ingenuity Pathway Analysis (IPAversion 21901358; release date December 9, 2014) to examine biological differences in salivary constituents revealed that neonatal saliva had unique gene transcripts related to embryonic development, hair and skin development and function, and organ development not found in adult subjects $(\mathrm{p}<0.01)$ ( Maron, unpubl.). These findings further support the unique properties of neonatal saliva.

In 2006, Kumar and colleagues (2006) refuted the notion that salivary RNAs were detectable in human saliva, stating that signals on microarrays and RT-qPCR amplification were caused by genomic DNA contamination. However, a series of published reports have confirmed the presence of saliva mRNAs, leaving little doubt of their potential for enhancing and informing clinical care ( $\mathrm{Li}$ et al. 2004a; Hu et al. 2006, 2008; Park et al. 2007; Maron et al. 2010; Lee et al. 2011). Extracted mRNAs have been applied to multiple downstream transcriptomic platforms including microarrays ( $\mathrm{Li}$ et al. 2004a), RT-qPCR (Maron et al. 2012b), and RNA sequencing (Spielmann et al. 2012). Each technique offers the investigator a slightly different approach to interrogate the transcriptome. Although microarrays and RNA sequencing (RNAseq) provide the opportunity to interrogate global gene expression, RT-qPCR can provide highthroughput analysis of targeted gene biomarkers. RNAseq has the added benefit of improving our understanding of transcriptional regulatory processes and alternative splicing, particularly in the setting of disease and disrupted development in the newborn. It should be noted, however, that despite available stabilizing agents designed to inhibit bacterial overgrowth, saliva is a mixed species flora that may interfere with sequencing readouts (Spielmann et al. 2012).

Applying knowledge about the salivary transcriptome to disease and development is the ultimate goal of research. In recent years, biomarkers associated with diverse medical conditions have been described. Importantly, these initial biomarker panels extend beyond oral cancer ( $\mathrm{Li}$ et al. 2004b; Brinkmann and Wong 2011) and autoimmune diseases that affect the oral cavity (e.g., Sjögren syndrome) (Miceli-Richard and Criswell 2014). Rather, emerging reports highlight the diagnostic potential of salivary transcriptomic analysis for identifying ovarian cancer (Lee et al. 2012), respectable pancreatic cancer (Zhang et al. 2010a), lung cancer (Zhang et al. 2012) breast cancer (Zhang et al. 2010b), and sleep deprivation (Seugnet et al. 2006), among others. Each of these studies highlights the potential for noninvasive salivary transcriptomic diagnostics to better identify systemic pathophysiology and disease in adult populations and laid the foundation for the integration and application of salivary transcriptomics into neonatal care.

\section{TRANSLATING THE TRANSCRIPTOME; NEONATAL APPLICATIONS}

In 2010, the first published report on neonatal salivary gene expression described the enor- 
mous amount of developmental information available in as little as $10 \mu \mathrm{L}$ of neonatal saliva (Maron et al. 2010). By analyzing saliva samples obtained serially from five premature infants from the time of birth to discharge, investigators were able to show that global developmental systems could be monitored in real-time. Following comparative whole-transcriptomic microarray analyses aimed at identifying genes that statistically significantly changed their expression pattern over time, the investigators reported that infants downregulated genes associated with embryonic, hematologic, and connective tissue development, while simultaneously up-regulating genes involved in organ and tissue development, as well as neurodevelopment and behavior as they matured (Maron et al. 2010). This discovery phase study was the first to use neonatal saliva to understand normal and potentially pathologic development at a molecular level. It ultimately generated important hypotheses about oral feeding readiness in premature neonates.

The determination of oral feeding maturity in the preterm infant population remains a clinical challenge because of neurodevelopmental complexity (Lau et al. 2000; Barlow 2009) and its dependence on subjective nursing assessment (Palmer et al. 1993). Current standards of care mandate the use of cue-based feeding protocols that ultimately lack the necessary specificity and sensitivity required to avoid placing infants at risk for choking, desaturation, and feeding aversion. Prior research has shown that for a newborn to successfully feed they must integrate numerous cranial nerves and muscles, along with their sensory, neurodevelopmental, and hunger signaling pathways (Barlow 2009; Maron et al. 2015). Interestingly, in the original neonatal salivary transcriptome study, computational and systems biology reviews of gene targets highlighted biological pathways associated with feeding maturity. Because each study subject learned to orally feed during his/her hospitalization, genes identified in the initial study mapped to biological processes involved in cranial nerve development, facial morphogenesis, neurodevelopment, and feeding behav- ior (Maron 2011). Within the feeding behavior category, salivary gene expression of the neuropeptide $\mathrm{Y} 2$ receptor (NPY2R) was subsequently shown to be a highly informative biomarker of feeding immaturity in this population (Maron et al. 2012b).

$N P Y 2 R$ is a well-described hypothalamic gene involved in hunger regulation (Naveilhan et al. 1999; Huang et al. 2008; Hunt et al. 2011). It is one of five known receptors for neuropeptide $Y$ (NPY). Unlike the other NPY receptors, NPY2R is the only gene in which expression must be down-regulated to increase hunger (Pjetri et al. 2012). In 2012, Maron and colleagues (2012b) showed that expression of NPY2R in neonatal saliva had a $95 \%$ positive predictive value for neonatal feeding immaturity. However, despite its strong positive predictive value, its negative predictive value was only $27 \%$. Thus, NPY $2 R$ represents just one of the biological processes required for successful oral feeding in the newborn.

To address this lack in knowledge, additional neonatal salivary gene expression microarray analyses were conducted on premature newborns during the learning process of oral feeding. Computational analysis and systems biology review of the microarray data identified a panel of genes $(n=24)$ that were involved in the diverse biological systems required in oral feeding maturation including sensory integration, facial development, neurodevelopment, hunger signaling, and digestive system development (Maron et al. 2015). A large prospective validation phase testing each of these biomarkers, alone and in combination, on 400 saliva samples resulted in the further identification of five genes (NPY2R, WNT3, PLXNA1, NPHP4, and $A M P K)$, that when combined with postconceptional age and sex had good accuracy at predicting feeding success in the newborn (area under the receiving operator curve $=0.78$ ). This research not only laid the foundation for the integration of salivary diagnostics into neonatal care, but clearly showed the utility of exploring the salivary transcriptome to further our understanding of evolving and potentially disrupted development in neonates (Fig. 1). 
J.L. Maron
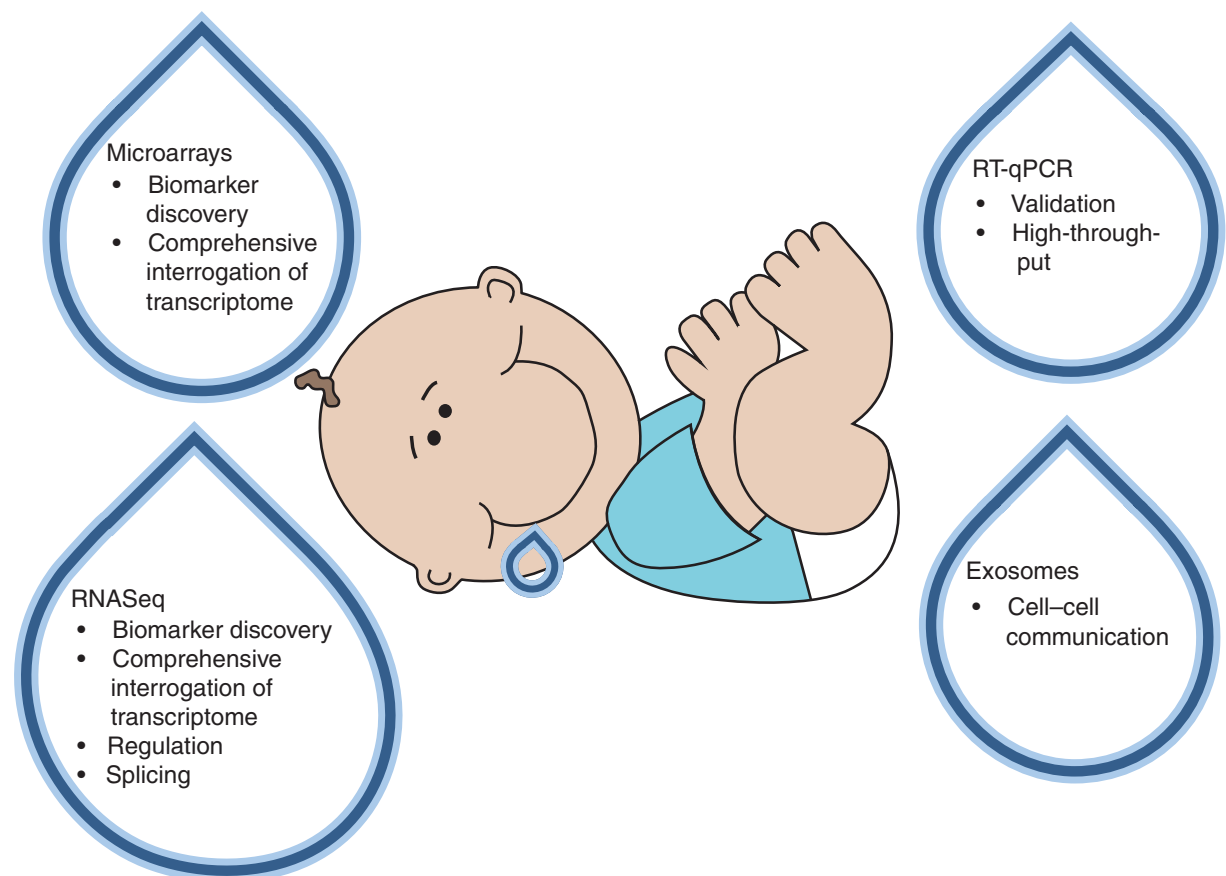

Figure 1. Neonatal salivary transcriptomics: Applications. (Modified from OpenClips image on Pixabay (C) 2015 [http://pixabay.com] under Creative Commons Deed CC0.)

FUTURE DIRECTIONS: RNA SEQUENCING, SALIVARY microRNAs, EXOSOMES AND BEYOND

Detection of mRNAs is only one component of salivary transcriptomics. RNA sequencing and exosome studies have also successfully been performed on adult human saliva samples (Palanisamy et al. 2010; Spielmann et al. 2012; Gallo and Alevizos 2013; Ogawa et al. 2013; Xie et al. 2013; Yang et al. 2014). Both RNA and exosome sequencing studies allow for simultaneous gene expression and transcriptomal regulatory mechanisms. Exosomes are small membrane vesicles that are known to contain miRNAs, proteins, mRNAs, noncoding RNAs (ncRNA), lipids, proteins, and cellular constituents (Thery et al. 2009). Emerging studies have shown that exosomes are essential for cell-to-cell communication throughout the body and play an important role in dissemination of disease (Alečković and Kang 2015), host-pathogen interactions (Schorey et al. 2014), and regenerative medicine (De Jong et al. 2014). Yang and colleagues (2014) have recently described the detection of tumor cell-specific mRNA in salivary exosome-like microvesicles of a novel human lung cancer xeongraft mouse model. Although similar studies in the newborn have yet to be performed, these technologies and approaches nevertheless hold the promise of furthering our understanding of aberrant development in this population.

\section{CONCLUSIONS}

Exploration of the salivary transcriptome is poised to enhance the ability to noninvasively monitor the developing, vulnerable newborn. Only simple modifications to existing protocols, combined with creative ways of collecting saliva are needed to gain access to real-time global and systemic developmental information that was never previously possible in the newborn. Technological advances are providing investigators with opportunities to not only interrogate whole exomes, but to further our understanding of transcriptional regulatory processes through RNA sequencing. These advances may ultimate- 
ly lead to novel treatment strategies. The detection of circulating exosomes containing regulatory miRNAs may also elucidate cell-to-cell trafficking and aid in diagnostics. Now is the time to integrate this technology into neonatology so that the most vulnerable of patients may benefit from noninvasive salivary diagnostics.

\section{ACKNOWLEDGMENTS}

The author would like to thank the hundreds of families cared for in the Tufts Medical Center Neonatal Intensive Care Unit and Mother Infant Unit who are helping to bring neonatal salivary transcriptomics to the bedside. Their willingness to participate in research is helping to transform neonatal care for the next generation.

\section{REFERENCES}

Alečković M, Kang Y. 2015. Regulation of cancer metastasis by cell-free miRNAs. Biochim Biophys Acta 1885: 24-42.

Balcarek KB, Warren W, Smith RJ, Lyon MD, Pass RF. 1993. Neonatal screening for congenital cytomegalovirus infection by detection of virus in saliva. J Infect Dis 167: 14331436.

Barlow SM. 2009. Central pattern generation involved in oral and respiratory control for feeding in the term infant Curr Opin Otolaryngol Head Neck Surg 17: 1871-1893.

Bianchi DW, Maron JL, Johnson KL. 2010. Insights into fetal and neonatal development through analysis of cell-free RNA in body fluids. Early Hum Dev 86: 747-752.

Brinkmann O, Wong DT. 2011. Salivary transcriptome biomarkers in oral squamous cell cancer detection. Adv Clin Chem 55: 21-34.

Cuevas-Córdoba B, Santiago-García J. 2014. Saliva: A fluid of study for OMICS. OMICS 18: 87-97.

De Jong OG, Van Balkom BW, Schiffelers RM, Bouten CV, Verhaar MC. 2014. Extracellular vesicles: Potential roles in regenerative medicine. Front Immunol 5: 608.

Dietz JA, Johnson KL, Wick HC, Bianchi DW, Maron JL. 2012. Optimal techniques for mRNA extraction from neonatal salivary supernatant. Neonatology 101: 55-60.

Gallo A, Alevizos I. 2013. Isolation of circulating micro RNA in saliva. Methods Mol Biol 1024: 183-190.

Hu S, Li Y, Wang J, Xie Y, Tjon K, Wolinsky L, Loo RR, Loo JA, Wong DT. 2006. Human saliva proteome and transcriptome. J Dent Res 85: 1129-1133.

Hu Z, Zimmermann BG, Zhou H, Wang J, Henson BS, Yu W, Elashoff D, Krupp G, Wong DW. 2008. Exon-level expression profiling: A comprehensive transcriptome analysis of oral fluids. Clin Chem 54: 824-832.

Huang X-F, Yu U, Li Y, Tim S, Deng C, Wang Q. 2008. Ventromedial hypothalamic NYP Y2 receptor in the maintenance of body weight in diet-induced obesity in mice. Neurochem Res 33: 1881-1888.

Hunt SC, Hasstedt SJ, Xin Y, Dalley BK, Milash BA, Yakobson E, Gress RE, Davidson LE, Adams TD. 2011. Polymorphisms in the NPY2R gene show significant associations with BMI that are additive to FTO, MC4R, and NpFFR2 gene effects. Obesity 19: 2241-2247.

Kumar SV, Hurteau GJ, Spivack SD. 2006. Validity of messenger RNA expression analyses of human saliva. Clin Cancer Res 12: 5033-5039.

Lau C, Alagugurusamy R, Schanler RJ, Smith EO, Shulman RJ. 2000. Characterization of the developmental states of sucking in preterm infants during bottle feeding. Acta Paediatr 89: 846-852.

Lawrence HP. 2002. Salivary markers of systemic disease: Noninvasive diagnosis of disease and monitoring of general health. J Can Dent Assoc 68: 170-174.

Lee Y-H, Zhou H, Reiss JK, Yan X, Zhang L, Chia D, Wong DTW. 2011. Direct saliva transcriptome analysis. Clin Chem 57: 1295-1302.

Lee YH, Kim JH, Zhou H, Kim BW, Wong DT. 2012. Salivary transcriptomic biomarkers for detection of ovarian cancer: For serous papillary adenocarcinoma. J Mol Med (Berl) 90: 427-434.

Li Y, Zhou X, St John MA, Wong DT. 2004a. RNA profiling of cell-free saliva using microarray technology. J Dent Res 83: 199-203.

Li Y, St John MA, Zhou X, Kim Y, Sinha U, Jordan RC, Eisele D, Abemayor E, Elashoff D, Park NH, et al. 2004b. Salivary transcriptome diagnostics for oral cancer detection. Clin Cancer Res 10: 8442-8450.

Llena-Puy C. 2006. The role of saliva in maintaining oral health and as an aid to diagnosis. Med Oral Patol Oral Cir Bucal 11: E449-E455.

Loo JA, Yan W, Ramachandran P, Wong DT. 2010. Comparative human salivary and plasma proteomes. J Dent Re 89: $1016-1023$.

Maganano CL, Gardner JM, Karmel BZ. 1992. Differences in salivary cortisol levels in cocaine-exposed and noncocaine-exposed NICU infants. Dev Psychobiol 25: $93-$ 103.

Maron JL. 2011. Exploring the neonatal salivary transcriptome: Technical optimization and clinical applications. Clin Biochem 44: 467-468.

Maron JL, Johnson KL, Rocke DM, Cohen MG, Liley AJ, Bianchi DW. 2010. Neonatal salivary analysis reveals global developmental gene expression changes in the premature infant. Clin Chem 56: 409-416.

Maron JL, Dietz JA, Parkin C, Johnson KL, Bianchi DW. 2012a. Performing discovery-driven neonatal research by transcriptomic analysis of routinely discarded biofluids. J Matern Fetal Neonatal Med 25: 2501-2511.

Maron JL, Johnson KL, Dietz JA, Chen ML, Bianchi DW. 2012b. Neuropeptide Y2 receptor (NYP2R) expression in saliva predicts feeding immaturity in the premature neonate. PLOS ONE 7: e37870.

Maron JL, Hwang JS, Pathak S, Ruthazer R, Russell RL, Alterovitz G. 2015. Computational gene expression modeling identifies salivary biomarker analysis that predict oral feeding readiness in the newborn. J Pediatr 166: 282-288. 
J.L. Maron

Miceli-Richard C, Criswell LA. 2014. Genetic, genomic and epigenetic studies as tools for elucidating disease pathogenesis in primary Sjögren syndrome. Expert Rev Clin Immunol 10: 437-444.

Naveilhan P, Hassain H, Canals JM, Ekstrand AJ, Larefalk A Chhajilani V, Arenas E, Gedda K, Svensoon L, Thoren P, et al. 1999. Normal feeding behavior, body weight and leptin response require the neuropeptide Y Y2 receptor. Nat Med 5: 1188-1193.

Ogawa Y, Taketomi Y, Murakami M, Tsujimoto M, Yanoshita R. 2013. Small RNA transcriptomes of two types of exosomes in human whole saliva determine by next generation sequencing. Biol Pharm Bull 36: 66-75.

Palanisamy V, Sharma S, Deshpande A, Zhou H, Gimzewski J, Wong DT. 2010. Nanostructural and transcriptomic analyses of human saliva. PLoS ONE 5: e8577.

Palmer MM, Crawley K, Blanco IA. 1993. Neonatal oralmotor assessment scale: A reliability study. J Perinatol 13: $28-35$

Pandit P, Cooper-White J, Punyadeera C. 2013. High-yield RNA-extraction method for saliva. Clin Chem 59: 1118 1122.

Park NJ, Zhou X, Yu T, Brinkman BM, Zimmermann BG, Palanisamy V, Wong DT. 2007. Characterization of salivary RNA by cDNA library analysis. Arch Oral Biol 52: 30-35.

Pennisi E. 2005. A mouthful of microbes. Science 307: 18991901.

Pjetri E, Adan RA, Herzog H, de Haas R, Oppelaar H, Speirenburg HA, Olivier B, Kas MJ. 2012. NPY receptor subtype specification for behavioral adaptive strategies during limited food access. Genes Brain Behav 11: 105-112.

Schafer CA, Schafer JJ, Yakob M, Lima P, Camargo P, Wong DT. 2014. Saliva diagnostics: Utilizing oral fluids to determine health status. Monogr Oral Sci 24: 88-98.

Schorey JS, Cheng Y, Singh PP, Smith VL. 2014. Exosomes and other extracellular vesicles in host-pathogen interactions. EMBO Rep e201439363.
Seugnet L, Boero J, Gottschalk L, Duntley SP, Shaw PJ. 2006. Identification of a biomarker for sleep drive in flies and humans. Proc Natl Acad Sci 103: 19913-19918.

Spielmann N, Ilsley D, Gu J, Lea K, Brockman J, Heater S, Setterquist R, Wong DT. 2012. The human salivary RNA transcriptome revealed by massively parallel sequencing. Clin Chem 58: 1314-1321.

Thery C, Ostrowski M, Segura E. 2009. Membrane vesicles as conveyors of immune responses. Nat Rev Immunol 9: 427-434.

Tiwari M. 2011. Science behind human saliva. J Nat Sci Biol Med 2: 53-58.

Wong DT. 2007. Salivary Transcriptome. Clin Cancer Res 13: 1350.

Wong DTW. 2012. Salivaomics. JADA 143: 19S-24S.

Xie Z, Chen G, Zhang X, Li D, Huang J, Yang C, Zhang P, Qin Y, Duan Y, Gong B, et al. 2013. Salivary microRNAs as promising biomarkers for detection of esophageal cancer. PLoS ONE 8: e57502.

Yang J, Wei F, Schafer C, Wong DTW. 2014. Detection of tumor cell-specific mRNA and protein in exosome-like microvesicles from blood and saliva. PLoS ONE 9: e110641.

Zhang L, Farrell JJ, Zhou H, Elashoff D, Akin D, Park NH, Chia D, Wong DT. 2010a. Salivary transcriptomic biomarkers for detection of resectable pancreatic cancer. Gastroenterology 138: 949-957.

Zhang L, Xiao H, Karlan S, Zhou H, Gross J, Elashoff D, Akin D, Yan X, Chia D, Karlan B, et al. 2010b. Discovery and preclinical validation of salivary transcriptomic and proteomic biomarkers for the non-invasive detection of breast cancer. PLoS ONE 5: e15573.

Zhang L, Xiao H, Zhou H, Santiago S, Lee JM, Garon EB, Yang J, Brinkmann O, Yan X, Akin D, et al. 2012. Development of transcriptomic biomarker signature in human saliva to detect lung cancer. Cell Mol Life Sci 69: 33413350 . 


\section{$\&_{\mathrm{CSH}}^{\infty} \&$ Cold Spring Harbor

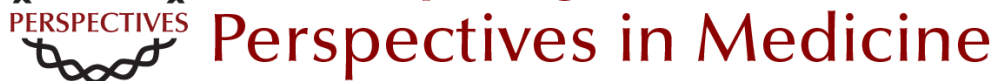

\section{The Neonatal Salivary Transcriptome}

Jill L. Maron

Cold Spring Harb Perspect Med 2016; doi: 10.1101/cshperspect.a026369 originally published online December 18, 2015

Subject Collection Molecular Approaches to Reproductive and Newborn Medicine

Intergenerational Transfer of Epigenetic Information in Sperm

Oliver J. Rando

Effects of Maternal Obesity on Fetal

Programming: Molecular Approaches Caterina Neri and Andrea G. Edlow

The Neonatal Salivary Transcriptome Jill L. Maron

The Role of Hox Genes in Female Reproductive Tract Development, Adult Function, and Fertility Hongling Du and Hugh S. Taylor

Molecular Cross-Talk at the Feto-Maternal Interface

Gendie E. Lash

Molecular Regulation of Parturition: A Myometrial

Perspective

Nora E. Renthal, Koriand'r C. Williams, Alina P. Montalbano, et al.

Genome-Wide Sequencing for Prenatal Detection

of Fetal Single-Gene Disorders

Ignatia B. Van den Veyver and Christine M. Eng

MicroRNA in Ovarian Biology and Disease

Lynda K. McGinnis, Lacey J. Luense and Lane K. Christenson
A Molecular Perspective on Procedures and Outcomes with Assisted Reproductive

Technologies Monica A. Mainigi, Carmen Sapienza, Samantha Butts, et al.

Whole-Exome Sequencing and Whole-Genome Sequencing in Critically III Neonates Suspected to Have Single-Gene Disorders

Laurie D. Smith, Laurel K. Willig and Stephen F. Kingsmore

Noninvasive Antenatal Determination of Fetal

Blood Group Using Next-Generation Sequencing Klaus Rieneck, Frederik Banch Clausen and Morten Hanefeld Dziegiel

Potential Uses and Inherent Challenges of Using Genome-Scale Sequencing to Augment Current Newborn Screening Jonathan S. Berg and Cynthia M. Powell

Molecular Regulation of Parturition: The Role of the Decidual Clock Errol R. Norwitz, Elizabeth A. Bonney, Victoria V. Snegovskikh, et al.

Molecular Mechanisms of Preeclampsia Tammy Hod, Ana Sofia Cerdeira and S. Ananth Karumanchi

Noninvasive Prenatal Screening for Genetic Diseases Using Massively Parallel Sequencing of Maternal Plasma DNA Lyn S. Chitty and Y. M. Dennis Lo

Confrontation, Consolidation, and Recognition: The Oocyte's Perspective on the Incoming Sperm David Miller

For additional articles in this collection, see http://perspectivesinmedicine.cshlp.org/cgi/collection/ 